\title{
Intensive Care Unit Use and Mortality in the Elderly
}

Wei Yu, PhD, Arlene S. Ash, PhD, Norman G. Levinsky, MD, Mark A. Moskowitz, MD

OBJECTIVE: To examine utilization and outcomes of intensive care unit (ICU) use for the elderly in the United States.

DESIGN: We used 1992 data from the Health Care Financing Administration to examine ICU utilization and mortality by age and admission reason for hospitalizations of elderly Medicare beneficiaries.

MAIN RESULTS: Use of the ICU was least likely for the oldest elderly overall ( $85+$ years, $21.1 \%$ of admissions involved ICU; 75-84 years, 27.9\%; 65-74 years, 29.7\%), but more likely during surgical admissions. Eighty-three percent of the Medicare patients who received intensive care survived at least 90 days. Of the oldest elderly, $74 \%$ survived. Even among the $10 \%$ most expensive ICU hospitalizations, $77 \%$ of all patients and $62 \%$ of those 85 years and older survived at least 90 days.

CONCLUSIONS: The likelihood of ICU use among these elderly decreased with age, especially among those 85 years or older. Diagnostic mix importantly influenced ICU use by age. The great majority of the elderly, including those 85 years and older and those receiving the most expensive ICU care, survived at least 90 days.

KEY WORDS: intensive care; hospital mortality; health services for aged.

J GEN INTERN MED 2000;15:97-102.

A ggressive medical care of the elderly, especially the oldest elderly (85 years and older), is an issue under vigorous discussion. The intensive care unit (ICU) is the locus of a large part of aggressive care, accounting for $20 \%$ to $30 \%$ of hospital care costs (approximately $\$ 62$ billion in 1992). ${ }^{1}$ No national population-based studies have examined patterns of ICU use by the elderly or clinical outcomes of such use. Studies using data from individual hospitals ${ }^{2-9}$ or states ${ }^{10,11}$ have reached different conclusions about clinical patterns of ICU use and subsequent mortality in the elderly.

In this article, we review these issues for all elderly Medicare-funded hospitalized patients during a study year, examining ICU use by clinical diagnosis and by age. We also determine death rates of patients in hospital and within 90 days of admission as a function of ICU use and age, paying special attention to the oldest elderly and to the most expensive $10 \%$ of hospitalizations and patients.

Received from the Health Care Research Unit, Section of General Internal Medicine, Evans Department of Medicine, Boston Medical Center, Boston, Mass (WY, ASA, MAM), and Boston University School of Medicine, Boston, Mass (NGL).

Address correspondence and reprint requests to Dr. Yu: Health Care Research Unit, Section of General Internal Medicine, Boston Medical Center, 720 Harrison Ave., Suite 1102, Boston, MA 02118 (e-mail: wyu@bu.edu).

\section{METHODS}

\section{Data}

Our analytic file was constructed using the Health Care Financing Administration's (HCFA) hospitalization and mortality records for its 5\% research sample of 1992 Medicare beneficiaries. We merged data from HCFA's Medicare Providers Analysis and Review Records (MEDPAR) and Annual Person Summary (APS) files.

The MEDPAR contains one record for each hospital stay including patient age, admission, and discharge date, all listed diagnoses and procedures, days and types of ICU care, and the diagnosis-related group (DRG). The DRG, a classification HCFA uses to determine the reimbursement for each hospital stay, is mainly a function of the principal diagnosis and the principal procedure. ${ }^{12}$ The MEDPAR file also lists nine types of ICU care and has a separate field for coronary care units. We included coronary care unit use under the generic rubric of ICU but excluded the 16 instances of pediatric ICU and 109 instances of psychiatric ICU from this study.

The 1992 APS contains one record for each person who received any Medicare-reimbursed services that year. These records contain demographic, enrollment, mortality, and annual utilization data, summarized in several categories.

\section{Study Sample}

From the APS file described above, we sampled Medicare beneficiaries older than 65 years of age, not in the end-stage renal disease (ESRD) program, within strata defined by levels of total cost. We took $100 \%$ of those in the highest percentile of total costs, $50 \%$ of those in the next four highest percentiles, $20 \%$ in the 75 to 94 percentiles, and $10 \%$ in the 0 to 74 percentiles. We then extracted all records of hospitalizations for these people in the MEDPAR file. This sampling method enabled us to focus on patients whose care was most expensive and yielded a study file with 175,723 hospital admissions experienced by 89,667 people. Among these people, 27,763 had at least one ICU admission during the year, generating a total of 54,774 distinct ICU admissions. We report all analyses weighted by the inverse of the sampling fraction to produce unbiased estimates for the entire aged, non-ESRD Medicare population.

\section{Intensive Care Unit Use and Mortality}

We examined numbers of admissions and frequency of ICU use and mortality in three age groups: 65-74, 75-84, and 85 years and older. We also examined outcomes of hospital care by age, using in-hospital death rates and 
deaths occurring within 90 days of admission. We did this separately for hospitalizations that included an ICU admission and for those that did not. We did not adjust for severity of illness when calculating mortality for hospitalizations that did and did not involve ICU care because our purpose was to observe differences in mortality between the two groups and how these differences are affected by age. In calculating the 90-day death rates for hospitalizations, we counted all admissions that occurred within 90 days of a death. For example, if a patient was hospitalized once on day 1 and again on day 40, then died on day 85, we counted each admission as leading to a death within 90 days. Thus, the patient in the example just given would have "died twice." This approach is the most inclusive way to measure mortality after ICU admission. The alternative of counting only one admission in a series within 90 days of death would understate the proportion of ICU admissions that can be characterized as unsuccessful in the sense that they did not lead to at least 90 days of survival.

We used DRG classifications to examine ICU utilization by "reason for admission." To provide focus, we selected the 20 most frequent DRGs among general hospitalizations for the elderly from the full list of 470 DRGs. We then added "closely related" DRGs to form 20 DRG groups. For instance, DRG 79, which is "respiratory infections and inflammations age $>17$ with complication and/or comorbidity," is among the 20 most common DRGs. We combined DRG 79 with DRG 80 ("respiratory infections and inflammations age $>17$ without complication and/or comorbidity") to create a DRG group called respiratory infection. After arranging the DRGs into groups, we dropped DRG 462 (rehabilitation) because it has only 1.3\% of ICU use, and added the next most common DRG group, coronary artery bypass graft (DRG 106 and 107). Admissions within these 20 DRG groups account for $50 \%$ of total hospital admissions and 60\% of total ICU admissions among elderly Medicare beneficiaries in our data.

To see if patients admitted within our 20 common DRG groups are similar to other hospitalized patients, we compared some demographics. The average age for persons with these 20 diagnoses was 77 years, compared with 76 years for all others. Women accounted for $58 \%$ of the most common admissions and 56\% of all other admissions. Among the most common admissions, the in-hospital mortality was $6.0 \%$ and the 90 -day mortality was $10.4 \%$. For the other hospital admissions, these figures were $5.1 \%$ and $9.0 \%$, respectively. Thus, the two groups appear to be comparable.

Because we wanted to know the outcome of the most costly ICU care, we separately examined 90-day mortality for the $10 \%$ of hospitalizations involving an ICU admission that were most expensive. We used the total amount paid by HCFA for each hospitalization to measure expense. This amount does not include payment for capital pass-through and indirect medical education, and excludes amounts paid by or on behalf of the patient. Although HCFA's expenditure is not an index of resource use for a hospitalization, it should be adequate to our purpose, which was only to determine death rates for the $10 \%$ most costly admissions. We examined age distributions and calculated 90-day death rates by age for this "most expensive" group.

We report rates per thousand to the nearest whole number, without confidence limits and significance tests. Because all rates are based on the experience of nearly 90,000 Medicare enrollees, the standard error of each reported rate is always very small in comparison with the rate. All differences in rates that we discuss are significant at the .01 level.

\section{RESULTS}

\section{General Intensive Care Unit Use}

In 1992, approximately 6.1 million aged Medicare beneficiaries (19\% of the study population) were hospitalized at least once, and approximately one quarter of those patients were admitted at least once to an ICU (Table 1). The 6.1 million people generated a total of 9.6 million admissions, $28 \%$ of which included ICU use. Comparing the three age categories, ICU use decreased with age, as a percentage both of hospitalized patients and of admissions.

\section{Intensive Care Unit Use by Type of Admission}

The likelihood of ICU use during an admission was higher for the 20 common DRG categories (33.4\% overall) than for other admissions (22\%) (Table 2). Within the common DRGs, the proportion of ICU use was higher when hospitalizations were for procedures (43.8\%) than for general medical conditions (30.5\%). Use of the ICU was uncommon (less than 15\%) when hip replacement or other hip and femur procedures were performed but occurred in half or more of hospitalizations for the other common procedures. Use of the ICU was frequent (39\%-80\%) for the DRGs that comprise general medical admissions for cardiac diagnoses (congestive heart failure, myocardial infarctions, angina pectoris, and cardiac arrhythmia), but

Table 1. Intensive Care Unit Use of Medicare Beneficiaries by Age

\begin{tabular}{lccccc}
\hline \hline & \multicolumn{2}{c}{ Hospitalized Patients } & & \multicolumn{2}{c}{ Admissions } \\
\cline { 2 - 3 } \cline { 5 - 6 } Age Group & $\begin{array}{c}\text { Number } \\
\text { (thousands) }\end{array}$ & $\begin{array}{c}\text { ICU } \\
\text { Use, } *\end{array}$ & & $\begin{array}{c}\text { Number } \\
\text { (thousands) }\end{array}$ & $\begin{array}{c}\text { ICU } \\
\text { Use, \% }\end{array}$ \\
\hline All ages $^{\dagger}$ & 6,142 & 26.8 & & 9,628 & 27.6 \\
$65-74$ y & 2,708 & 28.8 & & 4,232 & 29.7 \\
$75-84$ y & 2,361 & 27.2 & & 3,760 & 27.9 \\
$85+$ y & 1,073 & 20.8 & & 1,637 & 21.1 \\
\hline
\end{tabular}

* Patients who had at least one intensive care admission during the study year.

${ }^{\dagger}$ Due to rounding error, the number in the all-age group may not match the sum of three age groups. 
infrequent (6\%-28\%) during admissions for other common general medical diagnoses.

Figure 1 shows the pattern of ICU use by DRG group and age. For the procedural DRG groups other than hip and femur procedures, the percentage of admissions involving ICU use increased with age, especially for those 85 years and older. Among the 14 most common general medical reasons for admission (and for all other admissions; Fig. 1, last bar on right lower panel), the proportion of admissions involving ICU use declined with increasing age.

\section{Mortality of Hospitalizations With and Without Intensive Care}

We examined in-hospital and 90-day death rates following hospitalization in three groups: (1) all hospitalizations, (2) hospitalizations with ICU care, and (3) hospitalizations with no ICU care (Table 3). We also separately calculated these death rates by type of hospital admission (i.e., surgical vs medical admissions). Deaths were more likely for hospitalizations with an ICU admission and in- creased with age. However, the percentage by which ICU mortality exceeded non-ICU mortality was lowest in the oldest elderly. For 90-day mortality, for example, this increment was $40 \%$ for the younger age groups as compared with only $23 \%$ for those 85 years and older.

When mortality was separately calculated by type of admission, we found that deaths were less likely for surgical admissions than for medical admissions. For hospitalizations involving ICU care, the in-hospital mortality of surgical admissions was very close to that of medical admissions. But the 90-day mortality of surgical admissions was significantly lower than that of medical admissions. For hospitalizations involving no ICU care, both in-hospital and 90-day mortality were higher for medical admissions than for surgical admissions.

\section{Death Rates Following the Most Expensive Intensive Care Unit Hospitalizations}

Twenty percent of patients treated in the $10 \%$ most expensive hospitalizations involving ICU care died within 90

Table 2. Number of Hospital and Intensive Care Unit (ICU) Admissions of Medicare Beneficiaries by Admission Type

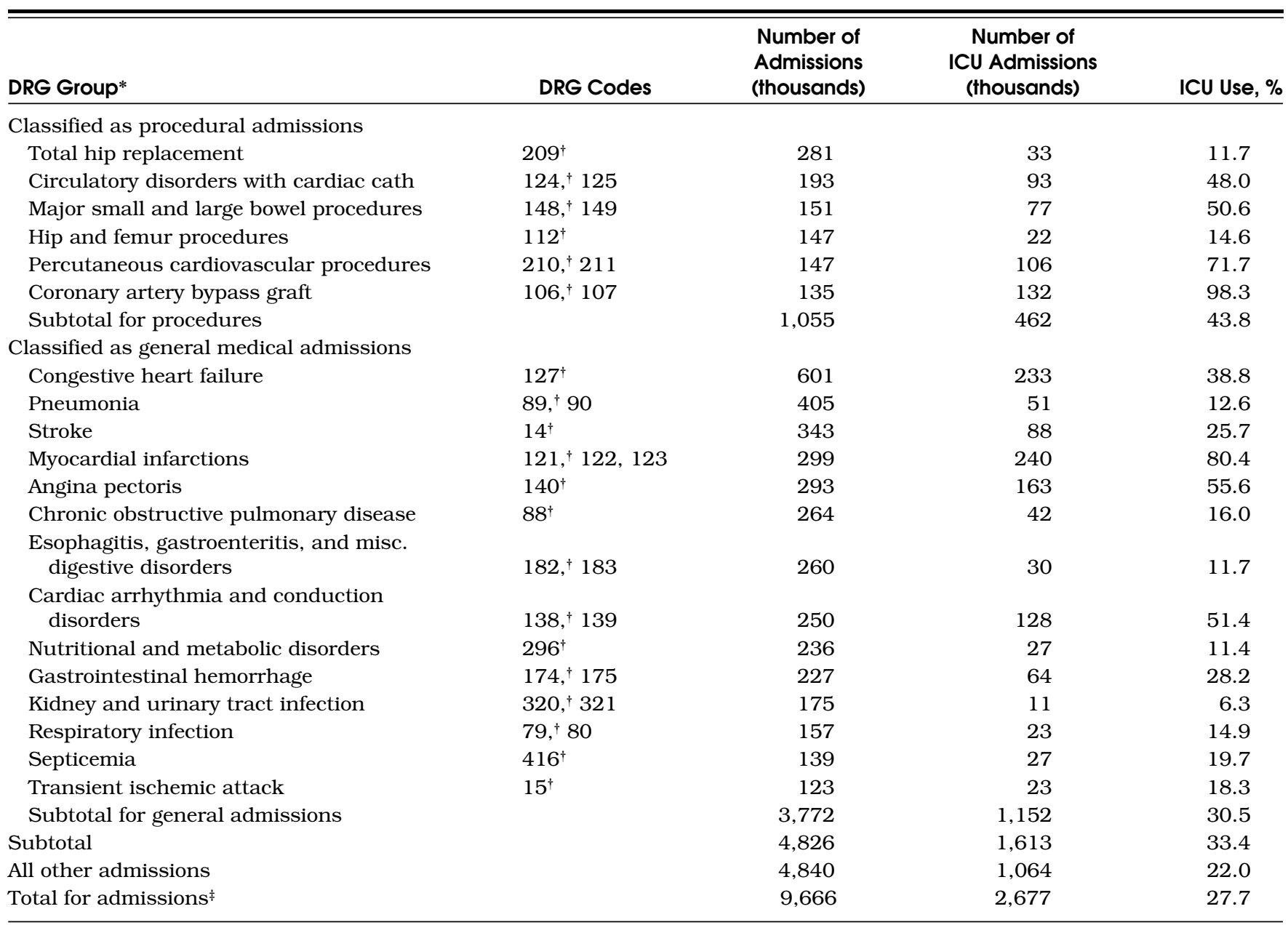

*Categories include all closely related diagnosis-related groups (DRGs) as listed. DRG groups are listed in order of the total number of admissions. † One of the 20 most common DRGs.

$\ddagger$ Due to rounding error, the total may not match the sum of subgoups. 


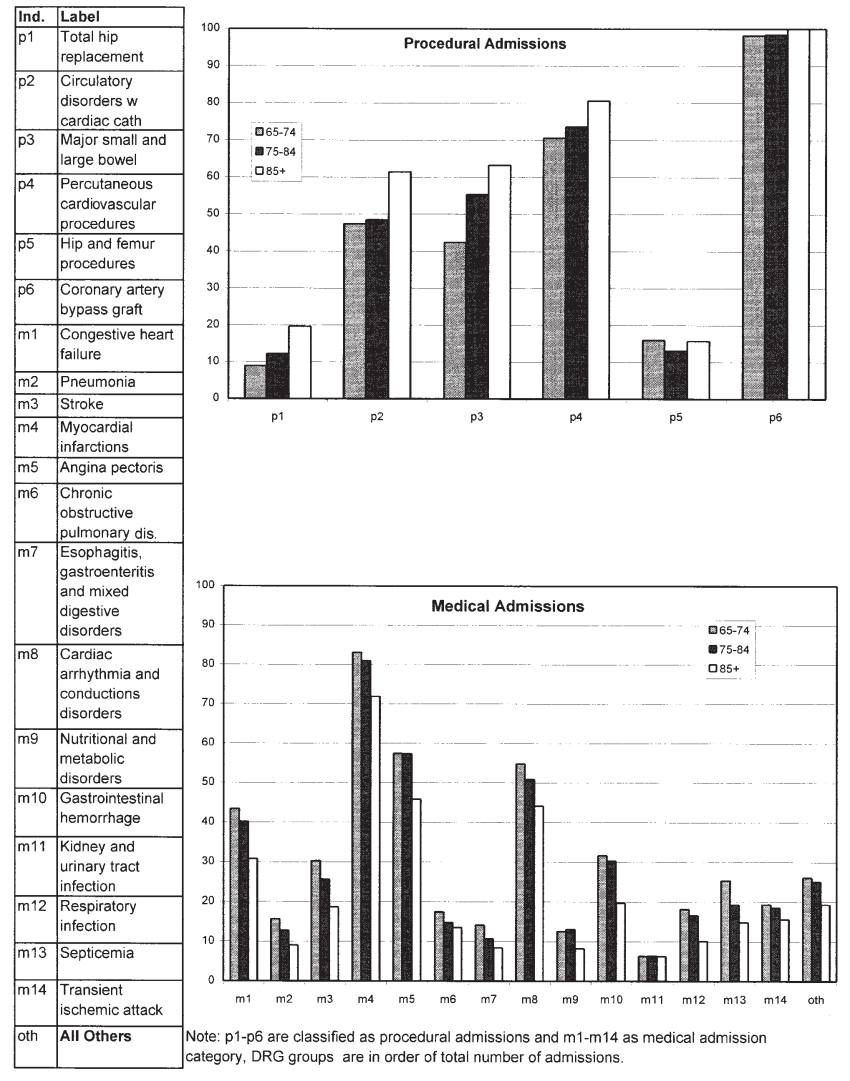

FIGURE 1. Percentage of admissions with intensive care unit utilization by admission type and age.

days. Thus, $2 \%$ of hospitalizations with ICU care were both very expensive and followed by death within 3 months. Mortality increased with age. However, even in the oldest group, a majority (62\%) survived. The oldest elderly accounted for $17.5 \%$ of all admissions, but a much smaller proportion of the most expensive admissions (6.4\%). The oldest patients also had the smallest percentage of most expensive hospitalizations with ICU care (65-74 years, $12.2 \%$; 75-84 years, $9.1 \%$; 85+ years, $4.9 \%$ ).

\section{DISCUSSION}

Although in general the patients aged 85 years and older were less likely to be admitted into the ICU than the patients between 65 and 84 years (Table 1), ICU use was more closely associated with medical conditions and treatments than with age (Fig. 1). When examining the common DRG groups, we observed a mixed pattern in ICU use with respect to age. The patients aged 85 years and older were less likely to receive intensive care in general medical admissions, but more likely to receive it in procedural and surgical admissions (Fig. 1). In another study from our group, patients aged 85 years and older were more likely than the other age groups to be hospitalized for general medical treatments and less likely to be admitted for major procedures. ${ }^{13}$ Thus, the major reason for lower ICU use among the group aged 85 years and older in the present study is probably that more of their hospital admissions were for general medical treatments, in which intensive care is less likely regardless of age. However, older patients admitted for procedures were usually more likely to use intensive care than younger patients (Fig. 1).

Our finding that the oldest elderly were less likely to be admitted for surgical procedures is generally consistent with a recent study using the data of five medical centers participating in the Study to Understand Prognoses and Preferences for Outcomes and Risks of Treatments (SUPPORT). That study shows that older age is associated with higher rates of decisions to withhold ventilator support, surgery, and dialysis even after adjustment for differences in patients' prognoses and preferences. ${ }^{14}$ In our data,

Table 3. Mortality Rates Following Hospital Admission by Age and Intensive Care Unit (ICU) Use

\begin{tabular}{|c|c|c|c|c|c|c|}
\hline \multirow[b]{2}{*}{ Admission } & \multicolumn{2}{|c|}{ Mortality-All Admissions } & \multicolumn{2}{|c|}{ Mortality with ICU Use } & \multicolumn{2}{|c|}{ Mortality without ICU Use } \\
\hline & In Hospital & 90 Days* & In Hospital & 90 Days* & In Hospital & 90 Days* \\
\hline \multicolumn{7}{|c|}{ Medical admissions } \\
\hline $65-74 y$ & 4.6 & 12.5 & 7.4 & 14.6 & 3.5 & 11.7 \\
\hline $75-84 y$ & 6.4 & 16.7 & 10.8 & 20.6 & 5.0 & 15.3 \\
\hline $85+y$ & 9.1 & 23.9 & 13.7 & 27.1 & 8.0 & 23.1 \\
\hline $65-74 y$ & 2.8 & 5.5 & 6.3 & 9.6 & 0.9 & 3.4 \\
\hline $75-84 y$ & 4.5 & 9.4 & 10.0 & 15.4 & 1.6 & 6.2 \\
\hline $85+y$ & 6.5 & 15.8 & 14.3 & 23.8 & 3.2 & 12.4 \\
\hline \multicolumn{7}{|c|}{ All admissions } \\
\hline All ages & 5.5 & 14.0 & 9.4 & 17.0 & 4.1 & 12.9 \\
\hline
\end{tabular}

*Death within 90 days of admission. 
however, the oldest elderly admitted for surgical admissions were more likely to be admitted to an ICU, suggesting no age bias against the oldest elderly in receipt of intensive care. A possible explanation is that physicians carefully selected only the most robust of the oldest group for surgery. This explanation is also consistent with our finding that patients admitted for surgical procedures were less likely to die (Table 3).

This observation may explain some conflicting findings about whether age determines ICU use for the elderly among prior studies. ${ }^{15}$ If a study is based on a specific medical condition, then the distribution of ICU use by age will be greatly influenced by the type of medical condition studied. Even if the age effect on ICU use is examined for a group of medical conditions, the aggregate result can be affected by the proportions of procedural and medical admissions selected. If a study sample is dominated by general medical admissions, it may be concluded that older patients use less intensive care; if the sample includes a high proportion of procedural admissions, the opposite conclusion may be drawn.

A major concern about ICU care is that this very expensive intervention may be overused, especially in the management of very elderly patients who are "at the end of their natural life spans." Cher and Lenert, ${ }^{10}$ for example, defined "potentially ineffective care" for Medicare patients who used an ICU as the concurrence of death within 100 days of hospital discharge and resource use above the 90th percentile. We did not estimate resource use but did evaluate the total amount paid by HCFA. Although our analysis differs in detail from their cost calculation, a general comparison is interesting. The 90-day death rate of the $10 \%$ most expensive ICU hospitalizations in the present study was $20 \%$, a $2 \%$ rate of potentially ineffective care for all Medicare hospitalizations with ICU care. This is much lower than the $4.8 \%$ estimate of the frequency of such an outcome for the California population Cher and Lenert studied. The difference is probably due to sample selection. The $4.8 \%$ rate of potentially ineffective care in their study is based on 15 DRG groups that have the highest 100-day mortality in ICU admissions, while the $2 \%$ rate in our study is based on all ICU admissions. In Cher and Lenert's study, the $4.8 \%$ of potentially ineffective care admissions accounted for $21.6 \%$ of total ICU resources. We cannot calculate the cost of ICU care from our data. However, the $2 \%$ of deaths among the most expensive hospitalizations in our study accounted for only $9.5 \%$ of the total amount paid by HCFA for all hospitalizations involving ICU care.

Clinical conclusions based on this type of analysis are speculative. Cost savings are possible only if the patients who die within a few months after the most expensive ICU care can be identified shortly after admission to hospital. There are as yet no accurate tools for identifying such patients prospectively without incurring the risk of denying appropriate care to the majority of elderly patients who are destined to survive for at least 3 months following ICU care. ${ }^{16-18}$ Of the most expensive hospitalizations in our study, over $60 \%$ of even the most elderly group survived, as did $84 \%$ of the 65 - to 74 -year-oldgroup. Moreover, only $5 \%$ of the oldest group received ICU care whose total hospital expenditure fell within the most expensive decile of ICU admissions. In addition, the percentage of the hospitalizations involving ICU care decreased with age, especially for the most elderly. Taken together, these observations suggest that only modest savings would accrue even if the ethically dubious decision were made to deny all ICU care for the very elderly; they also suggest that the term "potentially ineffective care" should be used with caution.

This study supports four specific conclusions. First, while the likelihood of ICU use following procedural admissions increased with age, the likelihood of ICU use following general medical admissions decreased with age. Second, although there was an association between age and ICU use, admission to the ICU was more highly associated with the admission diagnosis than with the age of the patient being admitted. Third, the likelihood of ICU use among the oldest hospitalized patients was lower than that for the younger population, since the oldest patients were less frequently admitted for procedural interventions, for which ICU use was highest. Finally, the substantial majority of all patients admitted to these ICUs survived for at least 90 days, including those 85 years and older and those whose hospital expenditures were in the most expensive decile of the Medicare payment for hospitalizations involving ICU care.

\section{REFERENCES}

1. Oye RK, Bellamy PE. Patterns of resource consumption in medical intensive care. Chest. 1991;99:685-9.

2. Layon AJ, George BE, Hamby B, Gallagher TJ. Do elderly patients overutilize healthcare resources and benefit less from them than younger patients? A study of patients who underwent craniotomy for treatment of neoplasm. Crit Care Med. 1995;23:829-34.

3. Cohen IL, Lambrinos J, Fein IA. Mechanical ventilation for the elderly patient in intensive care: incremental charges and benefits. JAMA. 1993;269:1025-9.

4. Mata GV, Fernandez R, Carmona A, et al. Factors related to quality of life 12 months after discharge from an intensive care unit. Crit Care Med. 1992;20:1257-62.

5. Roche VML, Kramer A, Hester E, Welsh CH. Long-term functional outcome after intensive care. J Am Geriatr Soc. 1999;47:18-24.

6. Chelluri L, Pinsky MR, Donohoe MP, Grenvik A. Long-term outcome of critically ill elderly patients requiring intensive care. JAMA. 1993;269:3119-23.

7. Rockwood K, Noseworthy TW, Gibney RTN, et al. One-year outcome of elderly and young patients admitted to intensive care units. Crit Care Med. 1993;21:687-91.

8. Castillo-Lorente E, Rivera-Fernandez R, Vazquez-Mata G. Limitation of therapeutic activity in elderly critically ill patients. Crit Care Med. 1997;25:1643-8.

9. Hanson LC, Danis M. Use of life-sustaining care for the elderly. J Am Geriatr Soc. 1991;39:772-7.

10. Cher DJ, Lenert LA. Method of Medicare reimbursement and the rate of potentially ineffective care of critically ill patients. JAMA. 1997;278:1001-7. 
11. Angus DC, Linde-Zwirble WT, Sirio CA, et al. The effect of managed care on ICU length of stay. JAMA. 1996;276:1075-82.

12. 3M Health Information Systems. DRGs: Diagnosis Related Groups. Definitions Manual, Version 10.0, Document No. 92-054 Rev. 00. 3M. St. Paul, Minnesota. 1992.

13. Levinsky NG, Ash AS, Yu W, and Moskowitz MA. The frequency of major procedures as reasons for hospitalization of the elderly. $\mathrm{J}$ Am Geriatr Soc. 1999;47:553-8.

14. Hamel MB, Teno JM, Goldman L, et al. Patient age and decisions to withhold life-sustaining treatments from seriously ill, hospitalized adults. Ann Intern Med. 1999;130:116-25.
15. Chelluri L, Grenvik A, Silverman M. Intensive care for critically ill elderly: mortality, costs, and quality of life. Arch Intern Med. 1995; 155:1013-22.

16. Lemeshow S, Teres D, Klar J, Arunin JS, Gehlbach SH, Rapoport R. Mortality probability models (MPM II) based on an international cohort of intensive care unit patients. JAMA. 1993;270:2478-86.

17. Civetta JM. Critical care: how should we evaluate our progress? Crit Care Med. 1992;20:1714-20.

18. Esserman L, Belkora J, Lenert L. Potentially ineffective care: a new outcome to assess the limits of critical care. JAMA. 1995; 274:1544-51.

\section{ANNOUNCEMENT}

\section{JGIM Website - Visit us online today!}

Please visit the JGIM World-Wide Website:

http://www.blackwellscience.com/journals 